\title{
Pattern Formation inside a Rotating Cylinder Partially Filled with Liquid and Granular Medium
}

\author{
Veronika Dyakova, Victor Kozlov, and Denis Polezhaev \\ Laboratory of Vibrational Hydromechanics of Perm State Humanitarian Pedagogical University, 24 Sibirskaya Street, \\ Perm 614990, Russia \\ Correspondence should be addressed to Denis Polezhaev; polezhaev@pspu.ru
}

Received 25 February 2014; Revised 31 July 2014; Accepted 27 August 2014; Published 11 September 2014

Academic Editor: Hamid Hosseini

Copyright (C) 2014 Veronika Dyakova et al. This is an open access article distributed under the Creative Commons Attribution License, which permits unrestricted use, distribution, and reproduction in any medium, provided the original work is properly cited.

\begin{abstract}
The paper focuses on the experimental study of the dynamics of liquid and granular medium in a rapidly rotating horizontal cylinder. In the cavity frame gravity field performs rotation and produces oscillatory liquid flow, which is responsible for the series of novel effects; the problem corresponds to "vibrational mechanics"-generation of steady flows and patterns by oscillating force field. The paper presents the initial results of experimental study of a novel pattern formation effect which is observed at the interface between fluid and sand and which takes the form of ripples extended along the axis of rotation. The initial results of experimental research of a novel effect of pattern formation at the interface between fluid and sand in the form of ripples extended along the axis of rotation are presented. The spatial period of the patterns is studied in dependence on liquid volume, viscosity, and rotation rate. The experimental study of long time dynamics of pattern formation manifests that regular ripples transform into a series of dunes within a few minutes or dozens of minutes. The variety of patterns is determined by the interaction of two types of liquid flows induced by gravity: oscillatory and steady azimuthal flows near the sand surface.
\end{abstract}

\section{Introduction}

The dynamics of liquid and granular medium in a rapidly rotating horizontal cylinder is experimentally studied. In a rapidly rotating cylinder liquid and granular medium coat the cylindrical wall under centrifugal force. The presence of gravity, acting in a direction perpendicular to the axis of rotation, results in a steady perturbation on the solid-body motion of liquid: central air column has its axis below the axis of the rotating cylinder. This leads to the fact that the liquid performs forced azimuthal oscillation in the cavity frame. Furthermore, in accordance with [1], the inertial waves with different azimuthal and axial wave numbers could be excited in the annular fluid layer. These waves were observed in the experiments at low rotation rates, that is, strong perturbing effect of gravity [2].

Inertial waves generate intensive oscillations in liquid and granular medium and fluidize the latter. It is known $[3,4]$ that oscillatory liquid flow near the flat sand bed provokes the onset of regular patterns in the form of ripples perpendicular to the axis of oscillation. In nature, sand ripples are formed on sea beds under back and forth motion of liquid. Although the formation of these patterns has been studied for a long time, the dominant wavelength selection mechanism is not clear yet. In various experiments on the pattern formation at the liquid-sand interface it is found that the spatial period of ripples $\lambda$ is proportional to the amplitude of fluid oscillations $b$ [5-8]. The surface waves also generate steady liquid flows in the direction of its propagation [9] and bring ripples on the sand bed to the continuous drift $[10,11]$. In nature, drift of patterns is caused by wind in a desert or by water in a river.

Thus, the discussed physical problem concerns the pattern formation under the integrated impact of oscillatory and unidirectional fluid flow. These flows are of importance in shorefaces, shelves, and so forth. Despite the importance of combined flows, our understanding of the bed forms generated by such flows is rather limited. In particular, because of complexity of continental shelves and the need 
for large laboratory flumes, the effect of surface waves is poorly understood. It is usually reported that sand dunes generated by tidal currents migrate downstream. Nevertheless, combined oscillatory and unidirectional fluid flows in wave tanks could induce both downstreaming and upstreaming sediment dunes [12]. In the experiments [13] authors studied the migration of ripples and estimated the drift velocity. In addition, the authors followed the dynamic evolution of ripples from the initial flat bottom configuration to the equilibrium state in a wave tank under propagating surface waves.

On the other hand liquid and granular medium are interrelated and liquid motion is influenced by distribution of granular medium. For instance, experiments [14] indicate that even small amount of heavy tracers can significantly modify the dynamics of liquid in a slowly rotating horizontal cylinder. The review [15] considers the results of theoretical and experimental studies of pattern formation of granular medium in rotating-drum flows. The most surprising result of the study is the discovery that the heavy [14] and light [16] particles and tracers of neutral buoyancy [17] display a tendency to axial segregation inside a rotating cylinder. The theory $[18,19]$ explains the segregation of neutrally buoyant particles by axial fluctuation of effective viscosity of liquid. However, the authors agree that the theory does not explain all the features of the phenomenon. The mentioned phenomenon is observed in the multiphase system of granular medium, liquid and gas inside a slowly rotating cylinder. In this case the centrifugal force is insufficient to centrifugate liquid and almost the entire volume of liquid and sand is at the cylinder bottom.

This paper concentrates on the dynamics of liquid and granular medium inside a rapidly rotating cylinder when liquid (and sand) forms an almost uniform annular layer. The advantage of this geometry is that it allows avoiding end effects, which strongly influence measurements in the experimental research. Depending on rotation rate axial or/and azimuthal fluid oscillations in the frame of rotating cylinder are excited under gravity. These oscillations can induce $3 \mathrm{D}$ relief on the sand bed. In the present paper we focus on the pure azimuthal oscillations and study regular ripples extended along the axis of rotation. The considered physical problem is somewhat similar to the stability problem for the interface between fluid and granular medium in an oscillatory cell [5] or under the impact of unidirectional shear flow in a stationary container [10].

The appearance of ripples on the sand bed is possible if only granules are able to move under fluid shear stress. The magnitude of shear stress is characterized by Shields number $\theta$, which is the ratio between the shear force on the grains and their apparent weight. The specific feature of the discussed problem is the variability of Shields parameter over the period of a cylinder rotation due to dependence of shear stress on the angle of sand-liquid interface inclination in the gravity field.

\section{Experimental Technique}

The experimental setup used in the investigation of pattern formation in the rotating cavity is shown in Figure 1. The

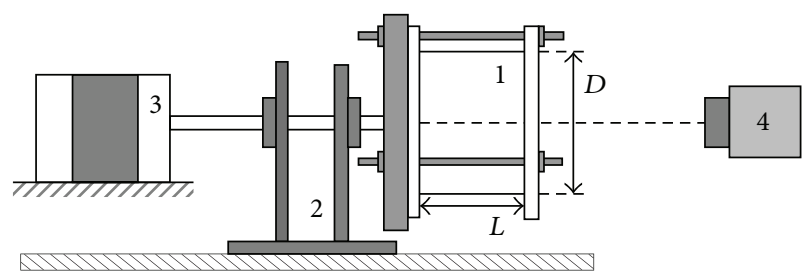

FIGURE 1: Sketch of experimental setup.

transparent circular cylinder 1 of length $L=7.8 \mathrm{~cm}$ and inner radius $R=D / 2=6.3 \mathrm{~cm}$ is fitted horizontally into ball bearings which are mounted onto a rigid stand 2 . The cylinder is driven at a constant rotation rate by the stepper motor 3 . The angular velocity of rotation $\Omega$ varies in the range from 0 to $50 \mathrm{~s}^{-1}$.

At the center of the rear wall of the cylinder there is a hole to fill it with liquid and granular medium. The experiments are conducted with water-glycerol mixtures; viscosity varies in the range from 1 to $10 \mathrm{cSt}$. The quantity of liquid is characterized by the relative filling $q=V / V_{0}$ ( $V$ is fluid volume, $V_{0}$ is a cavity volume) which varies in the range from 0.1 to 0.4 . The granular medium consists of glass spheres of diameter $d=0.15-0.25 \mathrm{~mm}$ and density $\rho_{s}=2.5 \mathrm{~g} / \mathrm{cm}^{3}$; mass $m$ of granular medium equals 125 or $250 \mathrm{~g}$.

Images of the cell are recorded via digital photo camera Nikon D40 4 positioned facing a transparent side wall. In order to provide high quality images of the liquid and sand the cross-section of the cell is illuminated by a flexible LED tape placed around the cylindrical wall. These images are then analyzed in order to explore the dynamics of inertial waves on the free surface of liquid and spatiotemporal evolution of patterns at the liquid-sand interface. In certain cases it is required to measure the velocity of wave propagation and the free surface of liquid is illuminated by a stroboscope lamp.

Each experiment follows a similar protocol. The cylinder is set in a rapid rotation to put fluid and sand into a state of solid-body rotation. Then, we decrease the rotation rate via one step-procedure to the required value of $\Omega$. The resulting fluid flow typically equilibrates within a few hundreds of the cylinder revolutions. Flow imaging is then carried out.

\section{Experimental Data}

3.1. Inertial Waves and Pattern Formation. While the cylinder is stationary, the liquid and sand rest in a pool on the bottom of the cylinder. When the cylinder rotates with low to moderate angular velocities its rising side drags out a thin film of liquid from the pool. The effect of gravitational force on the dynamics of rotating liquid is characterized by dimensionless acceleration $\Gamma=g / \Omega^{2} a$, where $a$ is the radius of free surface of liquid in the axially symmetric state of solid-body rotation, $g$ is the acceleration of gravity. When the cylinder rotates sufficiently fast, so that effect of gravitational force is small compared to centrifugal force $\left(\Gamma<10^{-1}\right)$, the entire pool of liquid and granular medium redistribute themselves to form two almost uniform annular layers coating the entire inner 


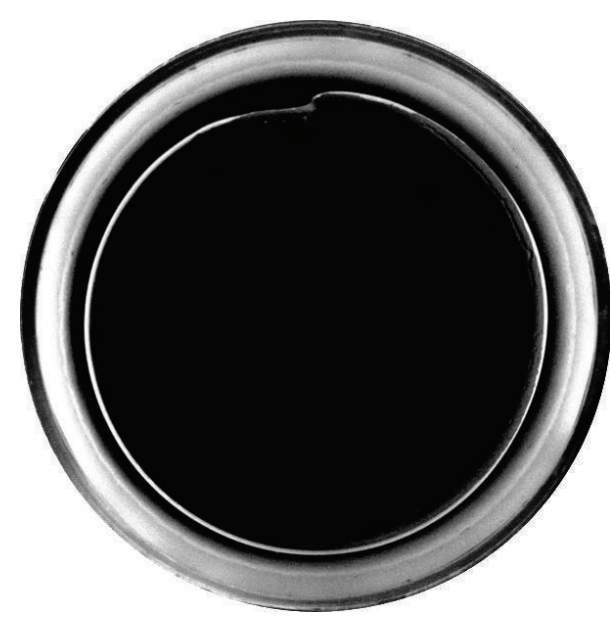

(a)

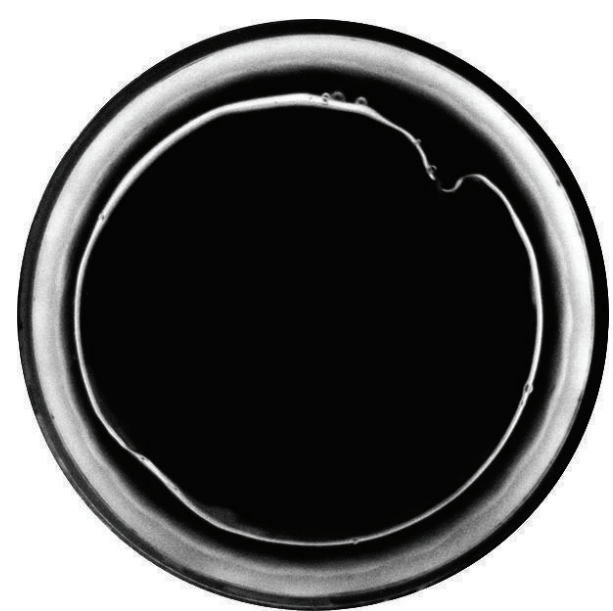

(b)

Figure 2: The azimuth inertial waves (front view): $l=1, q=0.236$, and $\Omega=32.0 \mathrm{~s}^{-1}$ (a) and $l=2, q=0.247$, and $\Omega=31.4 \mathrm{~s}^{-1}$ (b); $\nu=3.2 \mathrm{cSt}$ and $m=250 \mathrm{~g}$, hereinafter cylinder rotates counterclockwise.

surface of the cylinder. In accordance with [1], annular liquid layer is stable at $\Gamma<1 / 3$.

At the beginning of the experiment the cylinder rotates sufficiently fast and liquid and granular medium rest in the rotating frame. The decrease of $\Omega$ (increase of $\Gamma$ ) results in the raise of forced azimuthal liquid oscillations induced by gravitational force, and the oscillation frequency equals the cylinder rotation rate. At relatively large $\Gamma$ the oscillations with natural frequency could be excited. The natural frequency of oscillations depends on fluid volume [1]. In the studied range of relative filling $q$ inertial waves with nondimensional azimuthal wavenumber $l=1-5$ are observed, here $l \equiv 2 \pi a / L$, and $L$ is the azimuthal wavelength of a surface wave. For example, if we observe two wave crests (Figure 2(b)) then wavelength of azimuth wave $L=$ $\pi a$ and $l=2$. In the most studied range of $q=0.23-$ 0.29 the gravitational force induces two-dimensional waves with azimuthal wavenumber $l$ equaled to 1 or 2 (Figure 2). The wave profile depends on the azimuthal coordinate: the greatest deviation from the equilibrium position is on the top of the cell when the gravitational force and inertial force are opposite. The detailed study of inertial waves in the discussed configuration demonstrates that velocity of the wave propagation depends on the rotation rate $\Omega$ and wave amplitude increases with decrease of $\Omega$ [20].

While the rotation rate is high the amplitude of inertial wave is small and liquid-sand interface remains undisturbed. Then the rotation rate decreases gradually to obtain the critical value $\Omega_{c}$ at which the sand ripples arise. The experiments demonstrate that the time needed to reach the quasistationary state at the threshold of pattern formation is of the order of several minutes (or $\sim 10^{3}$ of the cylinder revolutions). At that rate, regular patterns rise in the form of squashed ripples extended along the axis of rotation (Figure 3). According to the observations of the sand bed, the first disturbances occupy only a few percents of the sand and spread everywhere within a few minutes; both height and azimuthal size of ripples change continuously until a quasistationary state is reached. The observations indicate that ripples are stationary in the rotating frame. In overcritical domain $\left(\Omega<\Omega_{c}\right)$ squashed ripples change to sinusoidal ripples extended along the axis of rotation (Figure 4 ). The observations show that the time which is necessary for the growth of regular sinusoidal patterns is shortened. Note that in the discussed experiments slopes of ripples are symmetrical.

A long-time behavior of sinusoidal ripples is complicated and will be discussed in Section 3.2. Here, we note that sinusoidal ripples retain a quasistationary form within a definite period of time and then transform into a series of irregular patterns. If the state of irregular patterns is reached and we further decrease the rotation rate then the sinusoidal ripples never reappear. Subsequent study of regular patterns is possible after the collapse of an annular layer and a further restart of the cylinder rotation.

Hereinafter we discuss the results of the experiments in the most studied range $0.23<q<0.29$. The experiments illustrate that wavelength in the quasistationary state $\lambda$ equals $2 \pi R_{s} / N\left(R_{s}\right.$ is the radius of flat sand bed and $N$ is the number of ripples) is independent of relative filling in the range $q=$ $0.23-0.29$ and decreases with increase of viscosity (Figure 5). The experimental data prove that ripples rise with decrease of $\Omega$.

Note that threshold angular velocity of pattern formation depends on liquid viscosity: in a less viscous liquid the ripples rise at a higher rotation rate. The decrease of angular velocity corresponds to the increase of dimensionless acceleration $\Gamma=$ $g / \Omega^{2} a$ and hence the increase of gravity influence. At a slow rotation annular liquid layer becomes unstable and collapses: regular patterns disappear.

3.2. Long Time Evolution of Ripples. The experimental data presented in Figure 5 are obtained within the first few minutes after the onset of ripples. According to the observations regular sinusoidal ripples retain quasistationary shape within 


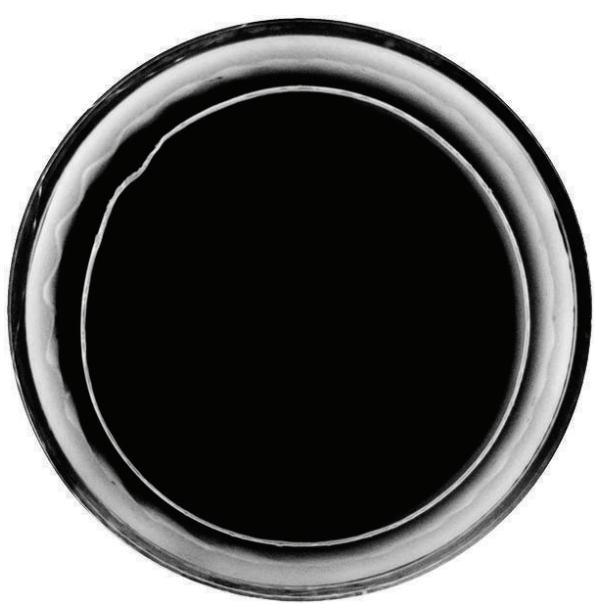

(a)

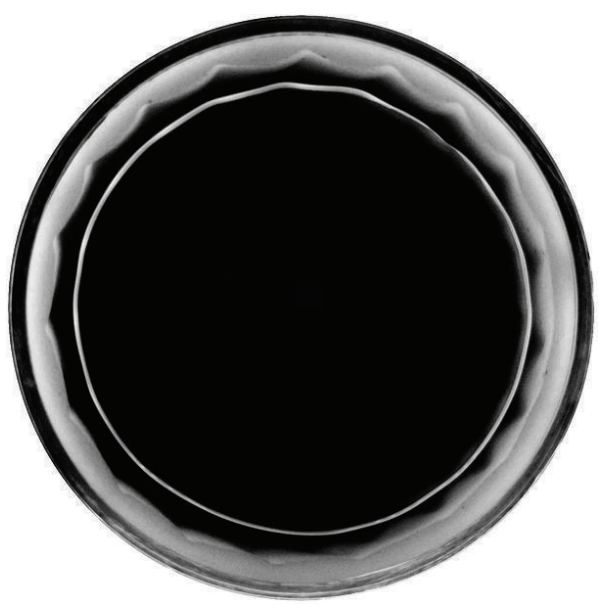

(c)

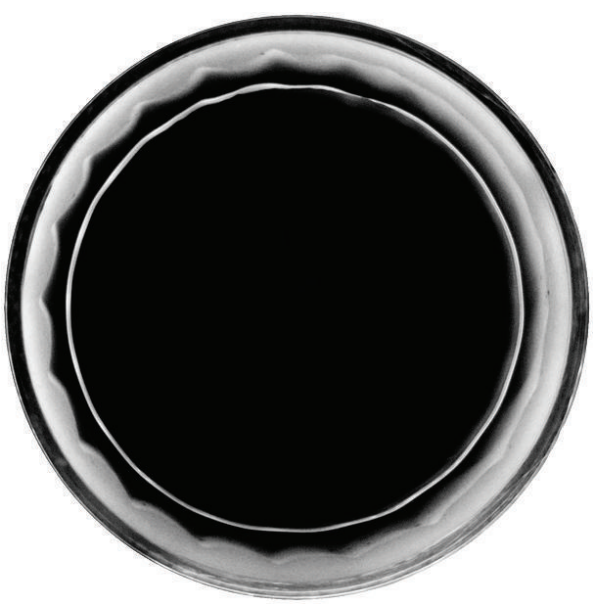

(b)

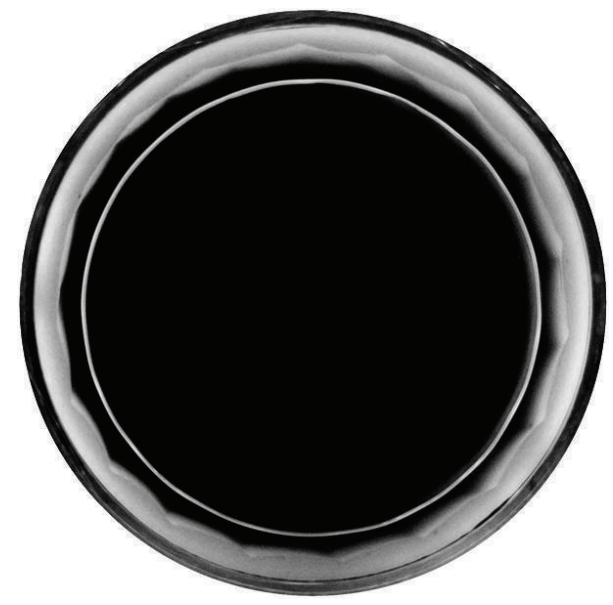

(d)

FIgURe 3: Time evolution of squashed ripples: $t=2,7,12$ and $50 \mathrm{~min}((\mathrm{a})-(\mathrm{d})) ; \Omega=35.6 \mathrm{~s}^{-1}, v=1.4 \mathrm{cSt}, q=0.215$, and $m=250 \mathrm{~g}$.

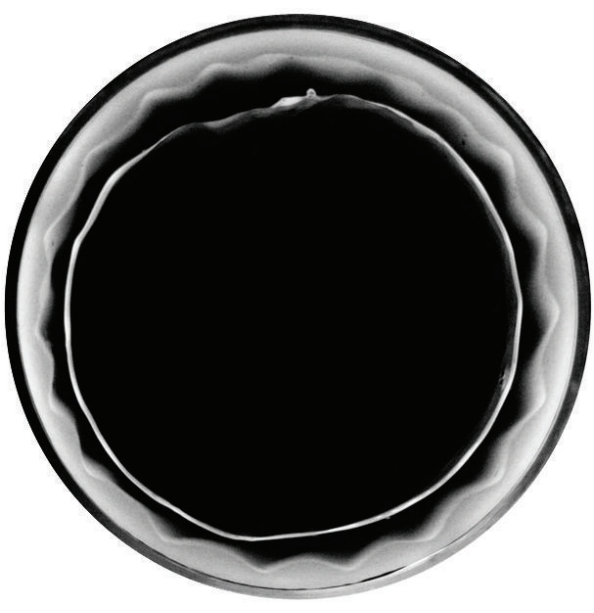

(a)

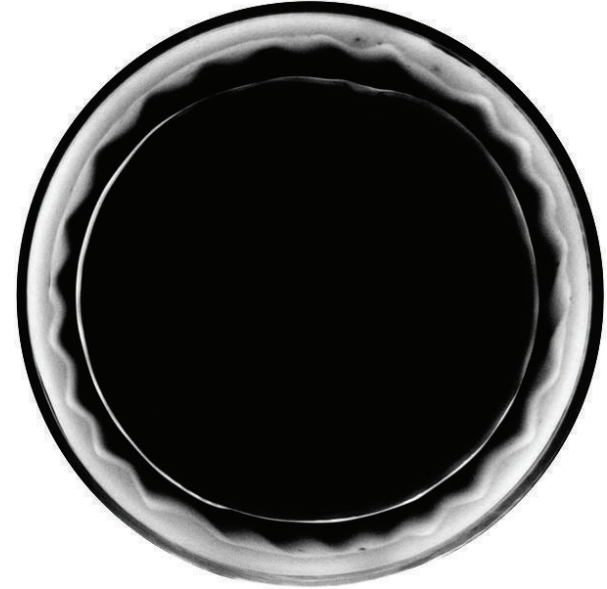

(b)

Figure 4: The sinusoidal ripples at $\Omega=33.9 \mathrm{~s}^{-1}$ (a) and $37.7 \mathrm{~s}^{-1}(\mathrm{~b}), q=0.257, v=1.4 \mathrm{cSt}$, and $m=250 \mathrm{~g}$. 


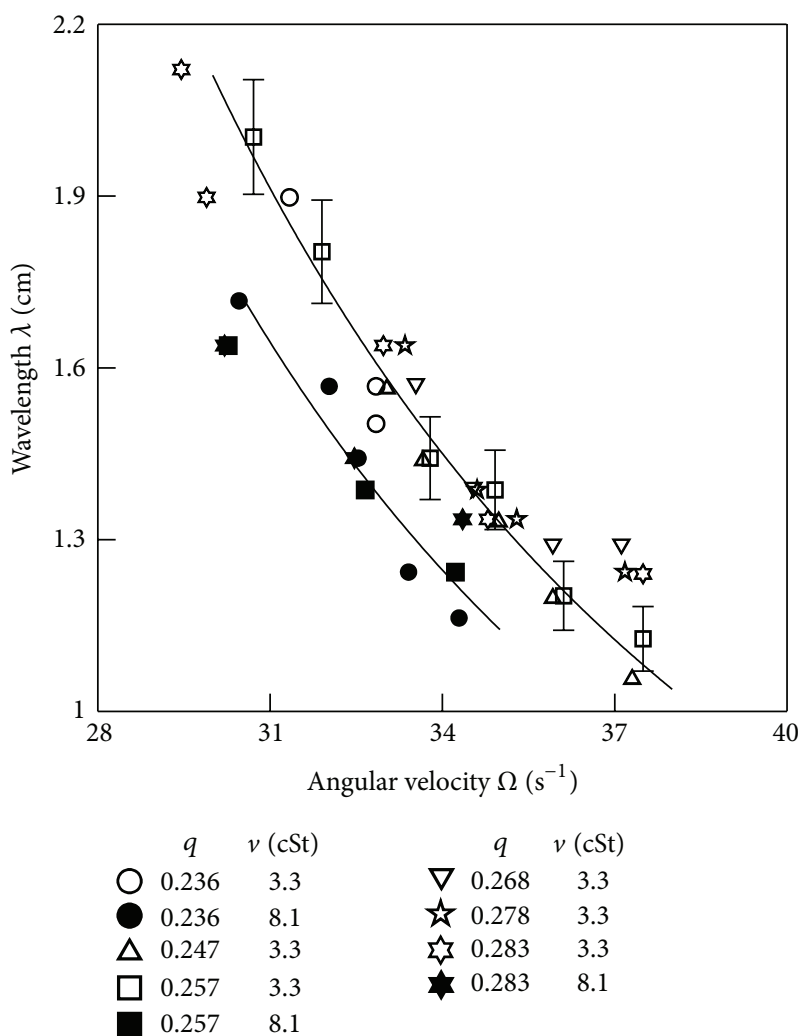

FIGURE 5: The wavelength of regular ripples versus angular velocity, $m=250 \mathrm{~g}$. The experimental data are obtained unless $T<10^{3}$. The typical error bars are shown.

a few minutes only and transform into a series of irregular dunes (Figure 6). A noteworthy fact is that in shallow liquid layers the free surface is curved due to the influence of the sand bed profile (Figure 6(b)).

A long time behavior of squashed and sinusoidal ripples is studied with the use of diagrams shown in Figures 7 and 8. Spatiotemporal diagrams illustrate that a long time evolution of sinusoidal ripples in overcritical domain (Figure 8) is much more complicated than the evolution of squashed ripples rising at the threshold of pattern formation (Figure 7). Together Figures 7 and 8 illustrate that in overcritical domain $\left(\Omega<\Omega_{c}\right)$ regular patterns rise much faster than near the threshold. Let us say regular sinusoidal ripples rise after approximately $30 \mathrm{~s}$ after the start of the experiment and regular squashed ripples appear within approximately $100 \mathrm{~s}$. Note that the squashed ripples are stationary in the rotating frame: negligible drift along the circumference is observed in the first minutes of the experiment only.

In order to distinguish between different phases of ripple formation we introduce dimensionless time $T=t / t_{0}$, here $t_{0}=\pi / \Omega$ is a period of the cylinder revolution. According to the diagram in Figure 7, at the threshold of appearance regular patterns keep form for at least $T=10^{4}$. On the other hand, regular ripples in overcritical domain keep form unless $T<10^{3}$ (Figure 8). Figure 8 manifests that sinusoidal ripples keep regular shape in the rotating frame; however, if $T$ is greater than $10^{3}$ then regular patterns transform into a series of irregular dunes (Figure 6(b)) and they start to drift. Note that both experiments prove that the wavelength of regular patterns is almost constant in time and $\lambda$ is allowed to be measured in the initial period of the experiments, that is, unless $T<10^{3}$. The experimental data in Figures 5 and 10 are obtained in the discussed period of time.

\section{Discussion}

The appearance of ripples on the sand bed is possible if only granules are able to move under fluid shear stress. The magnitude of shear stress is characterized by Shields parameter $\theta$, which is the ratio between the viscous shear force on the grains and their apparent weight.

We are able to estimate the Shields parameter $\theta$ for the granule on the sand bed in the rotating cylinder with fluid. In the rotating frame a granule is under the action of gravitational force, buoyant force, centrifugal force, and viscous shear force. In a rapidly rotating cylinder, gravitational force is much less than centrifugal force, dimensionless acceleration $\Gamma$ is of the order of magnitude $10^{-1}$ (Figure 9), and gravitational force can be neglected. Then, centrifugal force holds the granule fixed on the sand bed while the viscous shear stress is directed along the interface. In the said approximation, Shields parameter can be represented in the form

$$
\theta=\frac{v \rho_{l}(d u / d r)}{\left(\rho_{s}-\rho_{l}\right) \Omega^{2} R d},
$$

where $u$ is the velocity of shear fluid flow near the sand bed, $\rho_{l}$ is the density of the fluid, and $r$ is the radial coordinate. It should be remembered that shear flow is a superposition of forced oscillations and oscillatory flows induced by surface waves. The azimuthal velocity of forced oscillations in a rapidly rotating cylinder in the inviscid limit is obtained in [1]: $u=(1-q) g \cos \varphi / \Omega$, where $\varphi$ is the angle between the normal to the interface between liquid and sand and vertical axis. Note that the shear flow has a maximum velocity at the top and the bottom of the trajectory, that is, at $\varphi=0$ and $\pi$.

We are able to calculate the Shields parameter under the assumption that liquid performs forced oscillations. This assumption is valid at the threshold of ripple formation, when the amplitude of observed surface wave is small. Under gravity, liquid oscillates at the radian frequency equaled to the angular velocity of rotation: $\Omega_{\text {osc }}=\Omega$. Due to viscosity, a Stokes boundary layer of thickness $\delta_{S}=\left(2 v / \Omega_{\text {osc }}\right)^{1 / 2}$ is present at the interface between the fluid and the sand bed. Then, $d u / d r$ is of the order of magnitude $u / \delta_{S}$, and formula (1) takes the form

$$
\theta=\frac{(1-q) \nu \rho_{l} g}{\left(\rho_{s}-\rho_{l}\right) \Omega^{3} R \delta_{S} d}|\cos \varphi| .
$$

Formula (2) demonstrates variability of Shields number over the period of a cylinder rotation due to the shear stress dependency on the angle of sand-liquid interface inclination in gravity field. Then, we calculate the maximum value of 


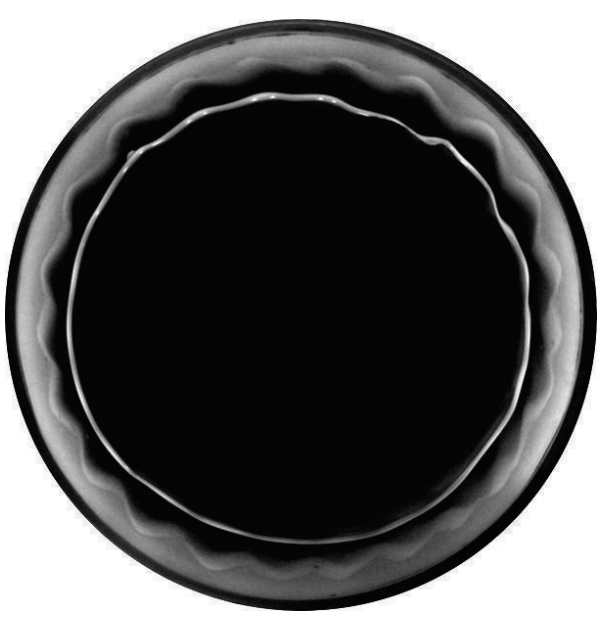

(a)

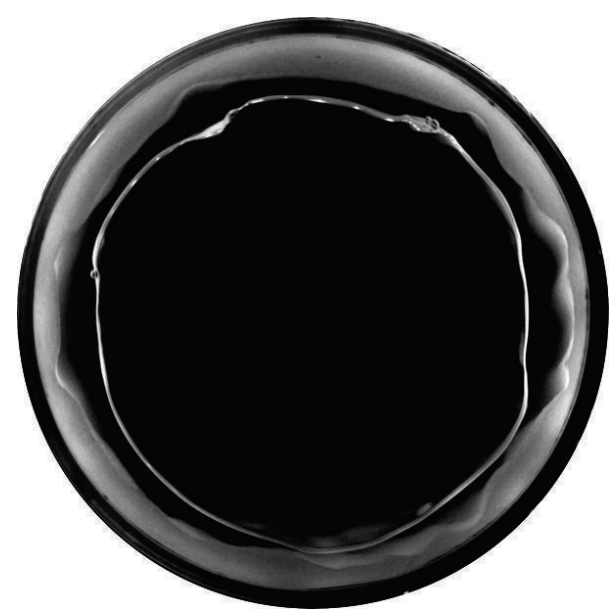

(b)

Figure 6: Time evolution of ripples at the liquid-sand interface: $t=1 \mathrm{~min}$ (a) and $60 \mathrm{~min}(\mathrm{~b}) ; q=0.257, v=3.2 \mathrm{cSt}, \mathrm{m}=250 \mathrm{~g}$, and $\Omega=33.0 \mathrm{~s}^{-1}$.

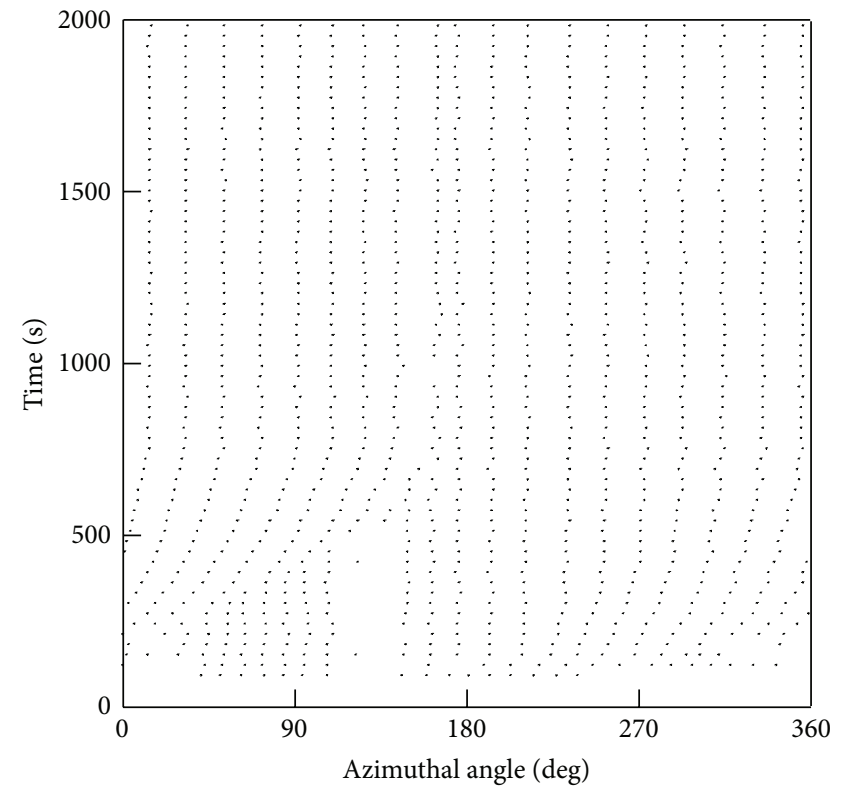

FIGURE 7: Spatiotemporal evolution of ripples: $q=0.216, v=1.4 \mathrm{cSt}$, $m=250 \mathrm{~g}$, and $\Omega=35.6 \mathrm{~s}^{-1}$; dots illustrate the positions of troughs between ripples. Azimuthal angle decreases in the direction of the cylinder rotation.

Shields parameter $\theta_{m}$, that is, at $\varphi=0$ or $\pi$. We can use values of $q, v$, and $\Omega$ obtained in Figure 5. In the case of forced oscillations, we should choose rightmost data in the plot $\lambda=\lambda(\Omega)$. According to the calculations, Shields parameter $\theta_{m} \approx 0.1$. A typical value of recognized Shields number in the literature for the onset of grain motion is $\theta=0.05$ [21]. Thus, in certain phases of the revolution granules are able to move along the sand bed and participate in the ripple formation.

Intensive fluid shear flow induces fluidization of the upper layer of the granular medium. The behavior of a fluidized granular medium is similar to that of liquid. The oscillatory

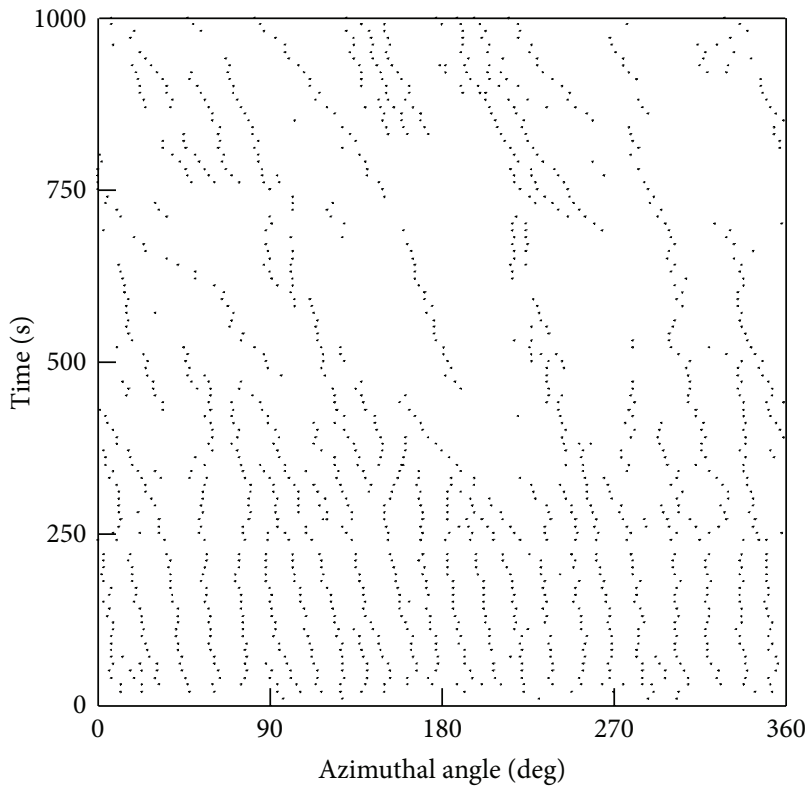

FIGURE 8: Spatiotemporal evolution of sinusoidal ripples: $q=0.262$, $v=1.3 \mathrm{cSt}, m=250 \mathrm{~g}$, and $\Omega=36.4 \mathrm{~s}^{-1}$; dots illustrate the positions of ripple peaks. Azimuthal angle decreases in the direction of the cylinder rotation.

motion in both media provoke Kelvin-Helmholtz instability [22] which is followed by the onset of regular ripples extended along the axis of rotation.

In various experiments on the pattern formation at the liquid-sand interface it is found that the spatial period of ripples $\lambda$ is proportional to the amplitude of fluid oscillations $b$; therefore $\lambda / b=$ const [5-8]. In the experiments focused on the ripple formation in a wave tank under the influence of combined fluid flows it is demonstrated that $\lambda / b=1.3$ [12]. The sustaining result is obtained in the problem of 


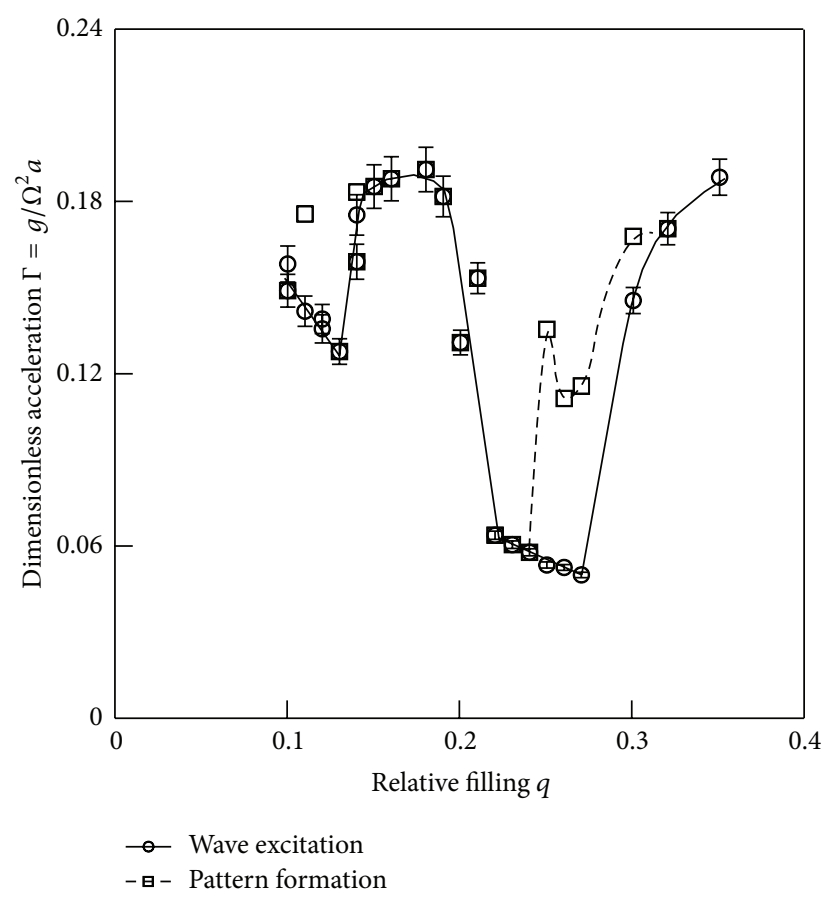

FIGURE 9: The thresholds of appearance of inertial waves and regular patterns; $v=1 \mathrm{cSt}$, mass of sand $m=125 \mathrm{~g}$.

the stability of the interface between sand and liquid in a cavity subjected to tangential oscillations (e.g., $[3,4]$ ).

We are able to estimate the parameter $\lambda / b$ at the threshold of ripple formation under the assumption that amplitude of an oscillatory flow induced by wave processes is small compared to the amplitude of forced oscillations. In overcritical region, surface waves are intensive and the used assumption is presumably invalid. Then, amplitude of fluid velocity could be calculated by using the formula $u=b \Omega_{\text {osc }}=(1-q) g / \Omega$. Here, we focus on the forced oscillations; therefore, frequency $\Omega_{\text {osc }}$ equals the rotation rate $\Omega$, and amplitude $b=(1-q) g / \Omega^{2}$. The experimental data at the threshold of ripple formation (rightmost data in Figure 5) demonstrate that parameter $\lambda / b$ is nearly 2 , which agrees qualitatively with data [12].

Let's consider the influence of wave processes on the pattern formation on the sand bed. The experimental data prove that evolution of sand ripples is influenced by liquid dynamics. According to the observations, ripples rise in the presence of inertial waves only. The observations are supplemented by the measurements of $\Gamma$ at the thresholds of inertial waves' excitation and rise of ripples in dependence on $q$ (Figure 9). The nonmonotonic dependence of $\Gamma$ on $q$ at the threshold of wave excitation is determined by the resonant intensification of liquid oscillations and is studied in detail in [2]. One could find that appearance of ripples follows the wave excitation; an exclusive behavior of ripples is found in the range $q=0.23-0.29$ : regular patterns rise at a slower rotation rate than inertial waves do. Our main aim here is to study fluid dynamics and pattern formation in this range of relative filling $q$.

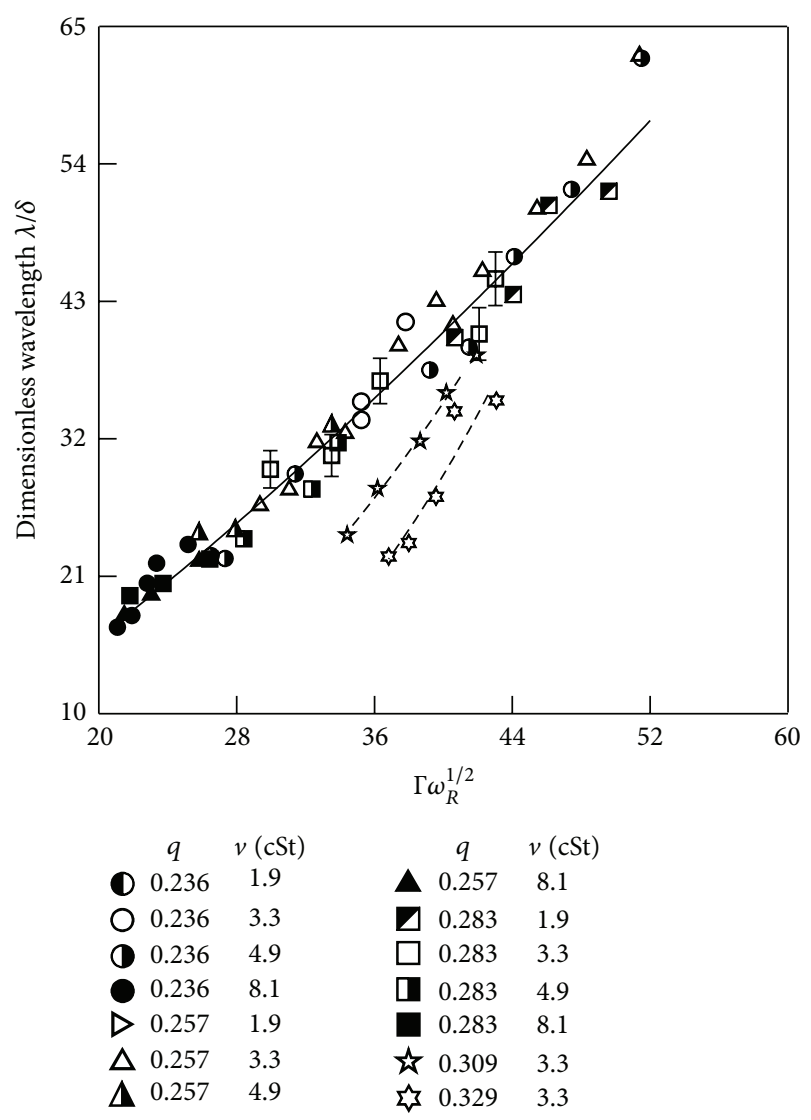

FIgURE 10: Dimensionless wavelength of regular ripples versus dimensionless parameter $\Gamma \omega_{R}{ }^{1 / 2}$; solid line corresponds to $\lambda / \delta \sim$ $\left(\Gamma \omega_{R}{ }^{1 / 2}\right)^{4 / 3}, m=250 \mathrm{~g}$. The experimental data are obtained unless $T<10^{3}$.

In the detailed study of inertial waves in a rotating cylinder [20] it is found that in relatively shallow layers of liquid $(q<0.24)$ gravity induces inertial waves with azimuthal wavenumber $l=1$ similar to the one shown in Figure 2(a), in deeper layers-waves with $l=2$ (Figure 2(b)). In the experiments with various masses of sand it is found that curve $\Gamma(q)$ shifts slightly along the axis " $q$ " and saves similarities with the one illustrated in Figure 9. So, near the relative filling $q=0.24$ we detect the change from inertial waves with $l=1$ to waves with $l=2$. The analysis of experimental data on the wavelength of regular ripples in the exclusive domain $q=0.23-0.29$ shows that $\lambda$ is determined by dimensionless parameter $\Gamma \omega_{R}^{1 / 2}$, where $\omega_{R}=\Omega R^{2} / v$ is the dimensionless velocity based on radius of a cylinder $R$ (Figure 10). The thickness of viscous boundary layer $\delta=$ $(2 v / \Omega)^{1 / 2}$ is introduced as the unit for the wavelength.

It is known [2,23] that in a horizontal cylinder partially filled with liquid inertial waves generate steady flow in the direction of its propagation. In the discussed experiments the gravity force produces azimuth wave and hence the liquid performs azimuthal motion. The velocity of liquid flow is proportional to $\Gamma^{2}$ [2]. While $\Gamma$ is small the intensity of azimuthal liquid flow is small compared to the oscillatory 
flow and regular patterns are stationary at the threshold of ripples growth (Figures 3 and 7).

The increase of $\Gamma$ (decrease of $\Omega$ ) is followed by intensification of azimuthal flow and appearance of migrating irregular dunes. The spatiotemporal diagram in Figure 8 indicates that dunes are not stationary in the rotating frame and drift in the azimuthal direction. It could occur if liquid and sand are involved in additional unidirectional flow. Apparently, we deal with the phenomenon similar to the drift of desert dunes under wind and bottom dunes under the influence of water currents. In the studied problem the unidirectional azimuthal current is generated by surface waves, and observed dunes migrate along the circumference. Surprisingly, the azimuthal drift of dunes in Figure 8 is opposite to the unidirectional fluid current. According to Figure 8, irregular dunes appear after approximately $T=$ $2 \times 10^{3}$ from the start of the experiment and migrate with velocity of about $10^{-1} \mathrm{~mm} / \mathrm{s}$. An effect of upstream-migrating sand is a rather odd phenomenon; nevertheless, it is found in the course of the field measurements [24] and observed in the laboratory experiments $[12,13]$. In the latter case, the sand dunes are generated under combined oscillatory and unidirectional fluid flow which is similar to the studied problem of pattern formation in a rotating cylinder under gravity. It is found that downstream and upstream-migrating dunes have different profiles and transition between different types of dunes depends on both oscillatory and unidirectional flow velocities. The effect of abnormal dune migration in a rotating cylinder with fluid under gravity is intriguing and requires further investigation of liquid flow near the sand bed.

The pattern formation in shallow $(q<0.23)$ and deep $(q>0.29)$ layers of liquid requires a detailed study. According to the observations in preliminary tests with shallow layers the ripples occupy only a few percents of sand surface after $T \sim 10^{4}$. In thick annular layers $(q>0.29)$, in the studied range of rotation rate we never obtain the solid-body rotation of fluid and observe surface waves and azimuthal liquid flows. These flows induce regular dunes on the sand bed which transform into irregular ripples after several thousands of the cylinder revolutions $\left(T>10^{3}\right)$. Apparently, the wavelength of dunes is determined by intensity of unidirectional azimuthal flow rather than oscillatory flow. Therefore, results of measurements of wavelength in the range $q>0.29$ do not agree with empirical law $\lambda / \delta \sim\left(\Gamma \omega_{R}^{1 / 2}\right)^{4 / 3}$ in Figure 10.

\section{Conclusions}

The dynamics of liquid and granular medium in a rapidly rotating horizontal cylinder is experimentally studied. It is found that axisymmetrical sand bed is unstable to the onset of regular patterns in the form of ripples extended along the axis of rotation. The pattern formation is associated with the excitation of inertial surface waves and corresponding steady flows caused by external force field; thus the phenomenon belongs to "vibrational mechanics." In nature, these flows are of importance in shorefaces, deserts, shelves, and so forth. In the most studied range of liquid volume the wavelength of regular ripples is determined by dimensionless parameter
$\Gamma \omega_{R}^{1 / 2}$. The long time dynamics of ripples is studied and found to be complicated: ripples save form within a few minutes or dozens of minutes and transform to the series of irregular dunes. The transition between two modes is determined by excitation of the steady azimuthal flow generated by azimuth progressive waves. The series of dunes perform slow azimuthal drift; the nature of this phenomenon is not clear yet and further study of liquid velocity profile is required.

\section{Conflict of Interests}

The authors declare that there is no conflict of interests regarding the publication of this paper.

\section{Acknowledgments}

The work is done within the framework of Strategic Development Program of PSPU (Project 048-M) and partially supported by Ministry of Education of the Perm region (Project C26/625).

\section{References}

[1] O. M. Phillips, "Centrifugal waves," Journal of Fluid Mechanics, vol. 7, pp. 340-352, 1960.

[2] A. A. Ivanova, V. G. Kozlov, and A. V. Chigrakov, "Dynamics of a fluid in a rotating horizontal cylinder," Fluid Dynamics, vol. 39, no. 4, pp. 594-604, 2004.

[3] A. Stegner and J. E. Wesfreid, "Dynamical evolution of sand ripples under water," Physical Review E, vol. 60, no. 4, pp. R3487-R3490, 1999.

[4] A. A. Ivanova and V. G. Kozlov, "Sand-fluid interface under vibration," Fluid Dynamics, vol. 37, no. 2, pp. 277-293, 2002.

[5] M. A. Scherer, F. Melo, and M. Marder, "Sand ripples in an oscillating annular sand-water cell," Physics of Fluids, vol. 11, no. 1, pp. 58-67, 1999.

[6] R. A. Bagnold and G. Taylor, "Motion of waves in shallow water. Interaction between waves and sand bottoms," Proceedings of the Royal Society of London. Series A, Mathematical and Physical Sciences, vol. 187, no. 1008, pp. 1-18, 1946.

[7] P. Nielsen, "Dynamics and geometry of wave-generated ripples," Journal of Geophysical Research: Oceans, vol. 86, no. 7, pp. 64676472, 1981.

[8] G. Rousseaux, J. Kruithof, P. Jenffer, and J. E. Wesfreid, "Oscillation-induced sand ripples in a circular geometry," Physical Review E, vol. 78, no. 1, Article ID 016302, 2008.

[9] G. K. Batchelor, Introduction to Fluid Dynamics, Cambridge University Press, Cambridge, UK, 1967.

[10] A. Betat, V. Frette, and I. Rehberg, "Sand ripples induced by water shear flow in an annular channel," Physical Review Letters, vol. 83, no. 1, pp. 88-91, 1999.

[11] A. Betat, C. A. Kruelle, V. Frette, and I. Rehberg, "Long-time behavior of sand ripples induced by water shear flow," European Physical Journal E, vol. 8, no. 5, pp. 465-476, 2002.

[12] S. Dumas, R. W. C. Arnott, and J. B. Southard, "Experiments on oscillatory-flow and combined-flow bed forms: implications for interpreting parts of the shallow-marine sedimentary record," Journal of Sedimentary Research, vol. 75, no. 3, pp. 501-513, 2005. 
[13] C. Faraci and E. Foti, "Evolution of small scale regular patterns generated by waves propagating over a sandy bottom," Physics of Fluids, vol. 13, no. 6, pp. 1624-1634, 2001.

[14] O. A. M. Boote and P. J. Thomas, "Effects of granular additives on transition boundaries between flow states of rimming flows," Physics of Fluids, vol. 11, no. 8, pp. 2020-2029, 1999.

[15] G. Seiden and P. J. Thomas, "Complexity, segregation, and pattern formation in rotating-drum flows," Reviews of Modern Physics, vol. 83, no. 4, article 1323, 2011.

[16] P. J. Thomas, G. D. Riddell, S. Kooner, and G. P. King, "Fine structure of granular banding in two-phase rimming flow," Physics of Fluids, vol. 13, no. 9, pp. 2720-2723, 2001.

[17] M. Tirumkudulu, A. Tripathi, and A. Acrivos, "Particle segregation in monodisperse sheared suspensions," Physics of Fluids, vol. 11, no. 3, pp. 507-509, 1999.

[18] B. Jin and A. Acrivos, "Rimming flows with an axially varying viscosity," Physics of Fluids, vol. 16, no. 3, pp. 633-640, 2004.

[19] B. Jin and A. Acrivos, "Theory of particle segregation in rimming flows of suspensions containing neutrally buoyant particles," Physics of Fluids, vol. 16, no. 3, pp. 641-651, 2004.

[20] V. V. Dyakova and D. A. Polezhaev, "Waves in the system of granular medium, liquid and gas in a horizontal rotating cylinder," Convective Flows, vol. 6, pp. 155-167, 2013 (Russian).

[21] P. Nielsen, Coastal bottom Boundary Layers and Sediment Transport, World Scientific, River Edge, NJ, USA, 1992.

[22] L. D. Landau and E. M. Lifshitz, Fluid Mechanics, Second Edition: Volume 6 (Course of Theoretical Physics), ButterworthHeinemann, Oxford, UK, 1987.

[23] A. A. Ivanova, V. G. Kozlov, and D. A. Polezhaev, "Vibrational dynamics of a centrifuged fluid layer," Fluid Dynamics, vol. 40, no. 2, pp. 297-304, 2005.

[24] G. Besio, P. Blondeaux, M. Brocchini, and G. Vittori, "On the modeling of sand wave migration," Journal of Geophysical Research C: Oceans, vol. 109, no. 4, 2004. 

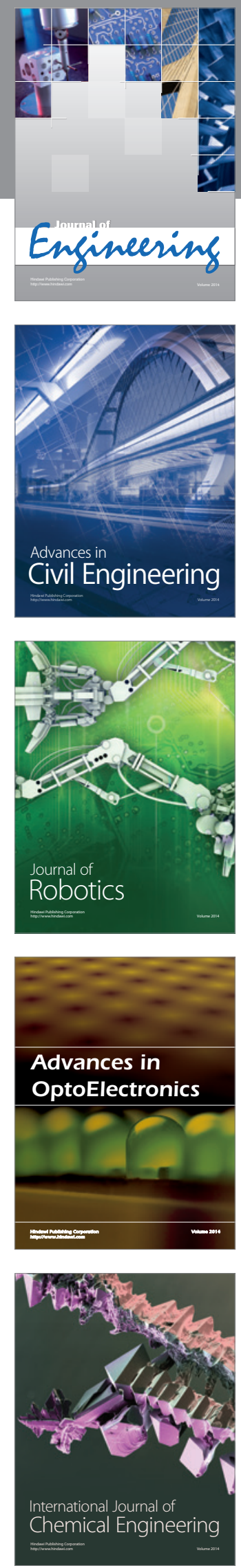

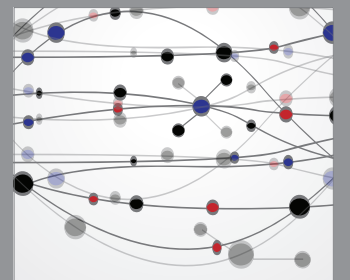

The Scientific World Journal
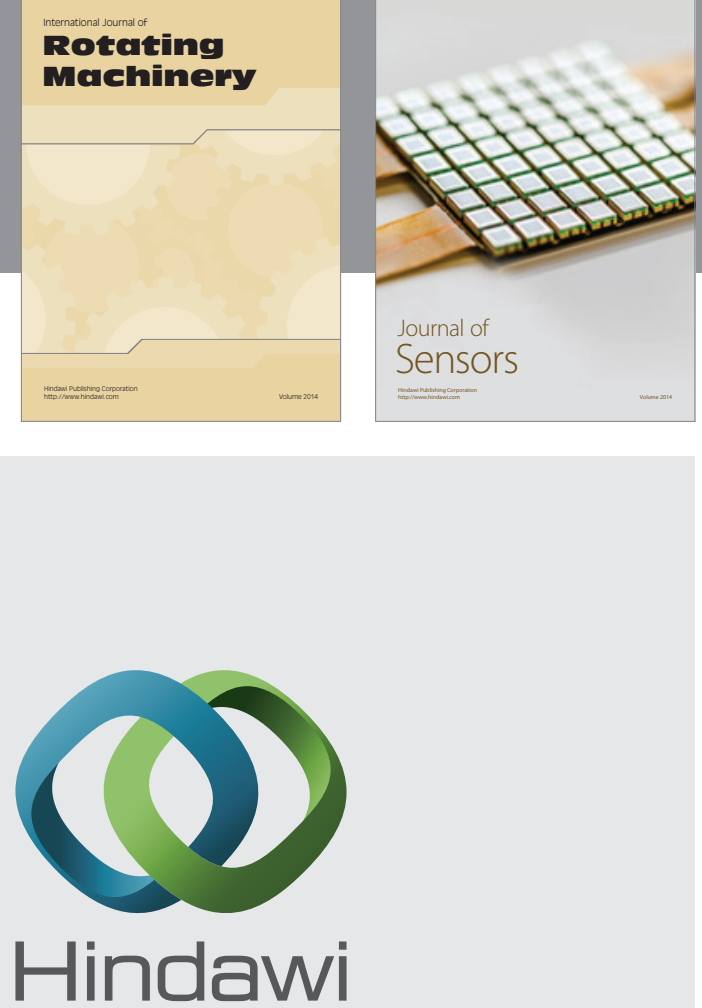

Submit your manuscripts at http://www.hindawi.com
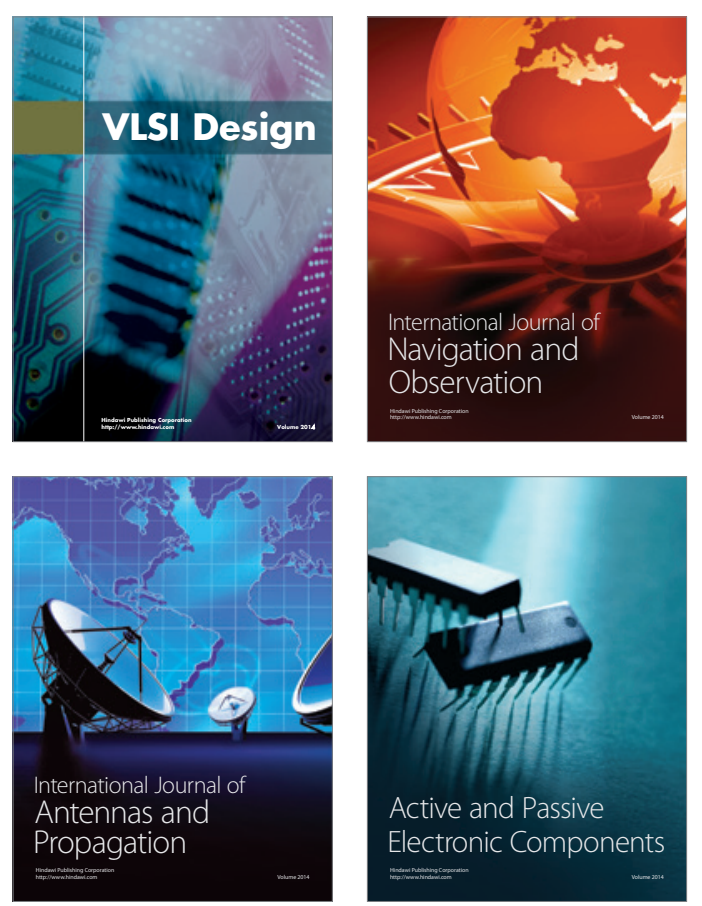
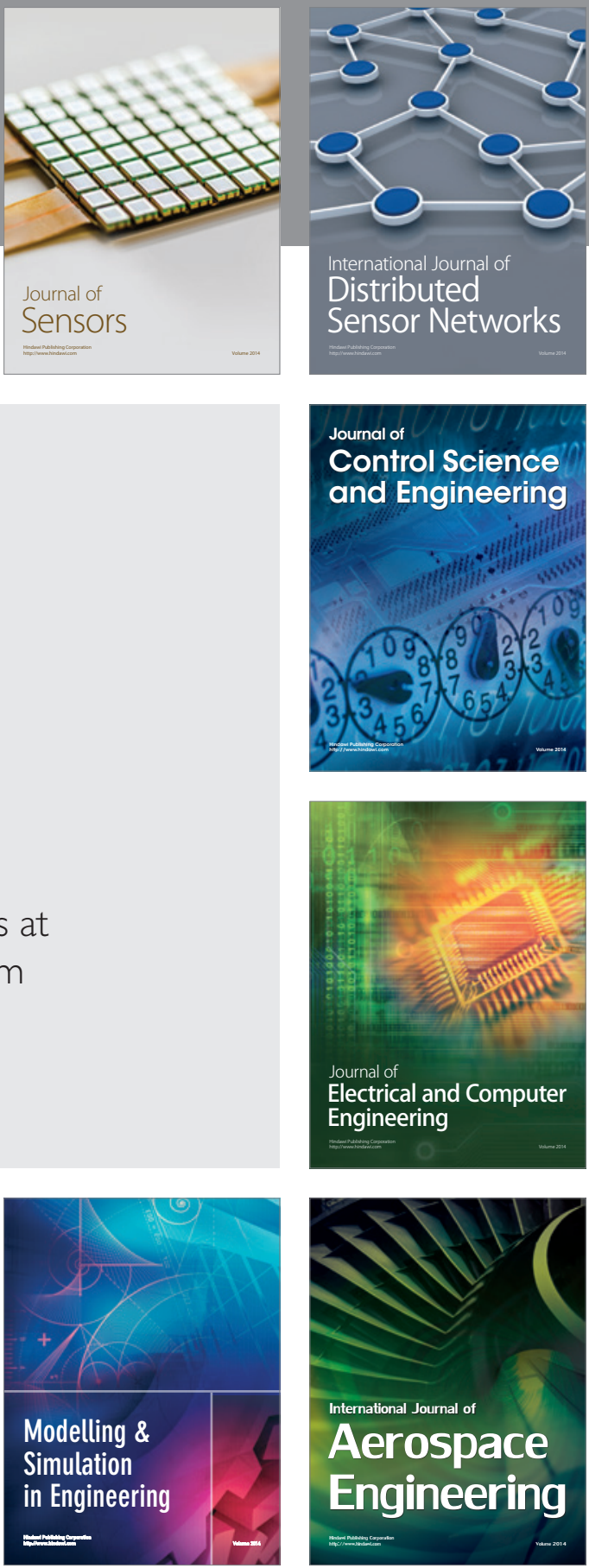

Journal of

Control Science

and Engineering
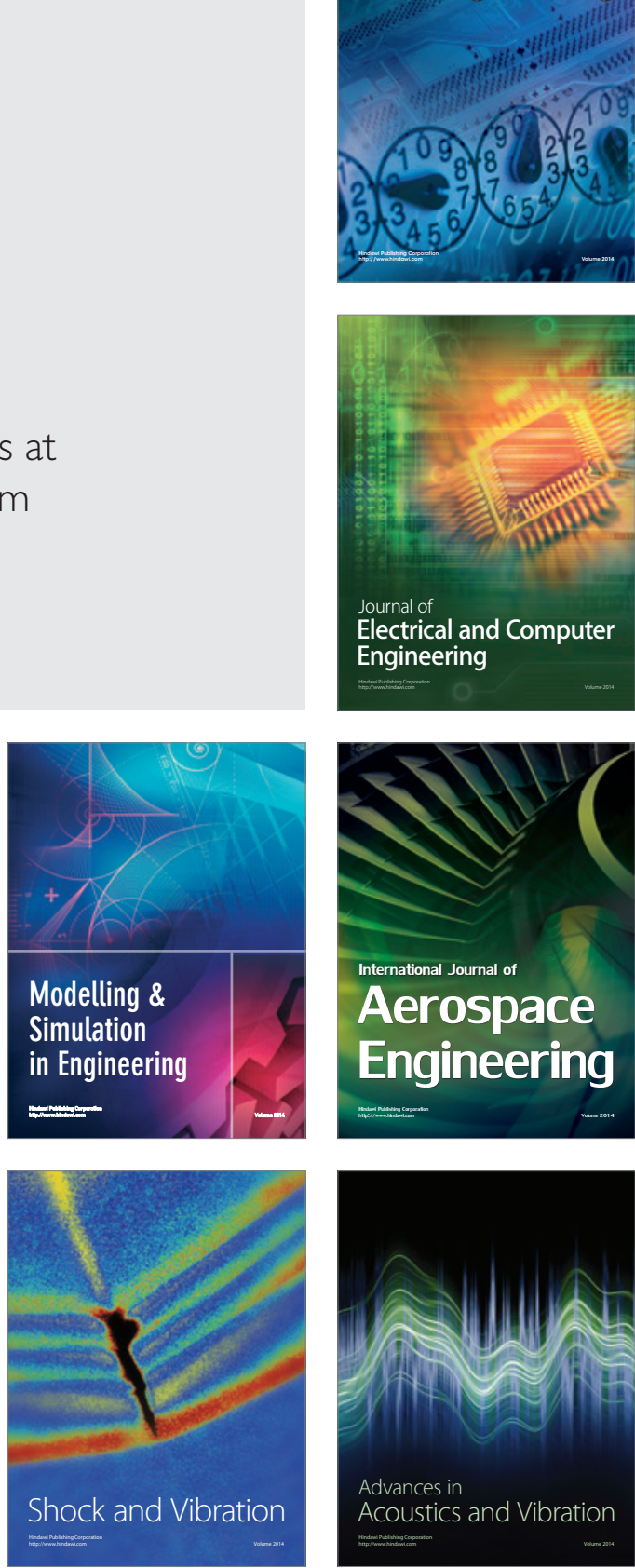